\title{
Prevalence of Different Salmonella serovars in B roiler Farms in Sharkia Governorate
}

Hamza M. I. Eid, Azza S. El-Demerdash, Nosayba M. Hassonna Dept. of Bacteriology, Mycology and Immunology, Fac. of Vet. Medicine, Suez Canal Univ., Egypt

Animal Health Research Institute, Dokki, Egypt

\begin{abstract}
:
Bacteriological examination of 300 samples from broiler internal organs (liver, cecum, unabsorbed yolk sac) from one day old chicks suffering from omphalitis and respiratory disorders after hatching, revealed 37 Salmonella isolates $12.3 \%$. Conventional methods for isolation and identification of Salmonella isolates from chicks showed that Salmonella appear as a colorless colony on Macconkeys agar medium. It gave the characteristics slightly transparent zone of reddish color with or without black center on XLD and colorless colonies with black center on SS agar medium. It appeared as Gram -negative straight rods, non-spore forming and arranged single in pairs and in groups .Moreover , Salmonella isolates were citrate test positive (blue color), urease test negative (yellow color)and they also gave acid but(yellow) and alkaline slant (red) with H25 production (black correlation on TSI agar medium. Serotyping of 37 Salmonella isolates by slide agglutination test using specific monovalent and polyvalent $\mathrm{O}$ and $\mathrm{H}$ Salmonella sera revealed eleven different Salmonella serotypes, with S. Enteritis as the most prevalent serotype (27.1\%) followed by S. Tamale (16.2\%)., S. Typhymurium and S. Kentucky (13.5\%) for each one, S. Anatum (10.8\%), S. Gallinarum 5.4\%, while S. Infntis, S. Bargny ,S. Takorade ,S. Heldberg and S. Larochelle were the last detected serovars $(2.7 \%)$ for each one. Anti-microbial sensitivity test revealed that most Salmonella isolates were sensitive to ciprofloxacin, sulfa methoxasole - trimethoprime, chlorumphenicol and gentamycin .while they were resistant to erythromycin,rifamycin ,amoxycillin /clavulinic acid ,colistin sulfate and cefotaxime .
\end{abstract}

Key words : Salmonella, serovars, Broiler. 
Introduction

Salmonella serovars are one of the primary foodborne pathogens. Poultry consumption is responsible for the majority of disease cases worldwide. The prevalence of virulence determinants among Salmonella serovars appears to be lacking in Egypt. Therefore, this study investigated the occurrence, antibiotic resistance patterns, and virulence gene profiling of Salmonella serovars in broilers Ammar et al., (2016).

Salmonella is an important pathogen for poultry production industry as well as for human due to zoonotic importance. It has more than 2600 identified serovars despite of this identification of Salmonella isolates into different serovars is critical for study of incidence and surveillance Sedeik et al., (2019).

The aim of work prevalence of different Salmonella Serovars in broiler farms in Sharkia Governorate

\section{Materials and Methods}

1.sampling

A total of 300 samples (liver, cecum and unabsorbed yolk sac; 100 from each organ) were collected from diseased and freshly dead broiler chickens.

Clinical tissue samples were collected aseptically to prevent cross contamination using sterile sampling materials (swabs, bags and syringes) and wearing disposable gloves. The samples were collected and transported in ice boxes with ice packs as early as possible to the laboratory for bacteriological examination.

2 Isolation of Salmonella and serotyping

The procedure for isolation and identification of Salmonellae were conducted according to ISO 6579 (2002) procedure. Suspected Salmonella colonies were confirmed serologically by Kauffman - White scheme (Kauffman, 1974) for the determination of Somatic (O) and flagellar $(\mathrm{H})$ antigens using Salmonella antiserum (DENKA SEIKEN Co., Japan). and biochemically by (TSI), Urea hydrolysis test, Lysine decarboxylation test, Indole production test and Citrate utilization test. The isolates were then serotyped by the Animal health research institute in Dokki -Giza. Only confirmed Salmonella were tested for their susceptibility to antimicrobial agents and the presence of the antimicrobial resistant genes.

3 Resistance to the antimicrobial agents

The antibiotic susceptibility was determined according to the recommendations set by the Clinical and Laboratory Standards Institute (Clinical and 
Laboratory Standards Institute, CLSI, 2007) for the disk diffusion technique. The antimicrobials concentrations tested were ampicillin $(10 \mu \mathrm{g})$, gentamicin $(10 \mu \mathrm{g})$, tetracycline $(30 \mu \mathrm{g})$ and sulfamethoxazole $\quad(25 \quad \mu \mathrm{g})$ (Oxoid, United Kingdom). The inhibition zones were measured and scored as sensitive, intermediate susceptibility or resistant according to the CLSI recommendations

\section{Results and Discussion}

Isolation and identification of Salmonella isolates:

\section{Colonial appearance:}

Salmonella grown onto MacConkey's agar medium gave pale colonies (non-lactose fermenter), subculturing from MaCconkey's agar onto xylose lysine desoxy cholate agar medium yielding colonies with aslightly transparent zone of reddish colour with or without black center.

\section{Biochemical identification:}

All Salmonella isolates were urea negative (yellow color),citrate positive (blue color),Salmonella isolates gave acid butt(yellow)and alkaline slant(red)with

$\mathrm{H} 2 \mathrm{~S}$

production(black coloration)on TSI agar medium.

Serotyping of Salmonellae isolates from chickens

Serotyping of 37 Salmonella isolates was applied by slide agglutination test using specific polyvalent "O" I, II, III and "H" Salmonella sera. Three different serotypes were identified among selected Salmonella isolates. The different serogroups were identified and Salmonella Enteritidis was the most prevalent onewith apercentage of $(27.1 \%)$ followed by $S$. Tamale (16.2\%), $S$. Typhymurium \& S.kentucky $(13.5 \%)$ for each one, $S$. Gallinarum (5.4\%), S. Apeyme (10.8\%), S.Larochelle, $S$. Takorade, S. Bargny, S. Infantis and $S$. Heldberg with percentage of $2.7 \%$ for each one.

\section{Prevalence of Salmonella in}

different organs of chickens in

El-Sharkia Governorate.

Thirty-seven Salmonella

isolates were recovered from

300 examined samples collected from chickens (12.1\%).

Salmonella was previously isolated from chicken by (Alshawabkeh and Yamani, 1996; Mohammed et al., 1999; Taha, 2002; Ahmed, 2003; Orji et al., 2005; Pieskusetal., 2006; Moawd, 2009; Maripandiand Ali 2010; Ahmed et al., 2016 and Ammar et al., 2019).

Nearly the same rates were obtained by Moawad (2009) who isolated Salmonella from chicken in Dakahlia Governorate with percentage $13.3 \%$ while higher rates were recorded by $\boldsymbol{A l}$ Shawabkeh and Yamani (1996) 
who recovered Salmonella with percentage $20.5 \%, 19 \%$ and $17.9 \%$, in layers, followed by broilers and breeders respectively.

Lower percentage rates than the previously mentioned were reported by Sadoma (1997) who isolated Salmonella from six out of $300(2 \%)$ cloal swabs collected from30 chicken farms at different localities in Garbia.

Moreover, Mohamed et al. (1999) isolated $S$. Typhymurium $S$. Anatum, and $S$.Pullarum from $2.5 \%$ of chickens and $4 \%$ of duck samples from fattening and laying farms in Kafr-Elshikh Governorate and Ahmed (2003) isolated Salmonella from chickens reared in rural village in Sharkia province with a percentage of $1.7 \%$.

This differences in prevalence rate may reflect considerable disparity in the sampling scheme, sample type, Salmonella detection protocol and geographic location.

Beside, eleven different serotypes were identified among selected isolates by slide agglutination test using polyvalent "O" 1,11,111 and $\mathrm{H}$ antisera.

The results of serological identification in present study detected eleven different serovars from 37 Salmonella isolates, $S$. Entertides predominated with higher percentage $(27.1 \%)$, while the remained isolates were serotyped as $S$. Tamale, $S$. Typhymurium, $S$. Kentucky, $S$. Apeyme, $S$. Gallinarum $S$. Infantis, $S$. Takorade, $S$. Heldberg, $S$. Bargany and $S$. Larochelle with percentages (16.2\%), (13.5\%), (13.5\%), $(10.8 \%), \quad(5.4 \%), \quad(2.7 \%)$, $(2.7 \%),(2.7 \%),(2.7 \%),(2.7 \%)$ respectively as in table (7).

In current study $S$. Entertides is the predominant serotype that go hand in hand with Sedeik et al. (2019).

Several authors isolate Salmonella Typhymurium from chickens at a higher rate than $S$. Enteritidis in India with a percentage of $18.10 \%$ and 9.87\% respectively (verma and Gupta, 1995) and in Singapore with apercentage of $23.8 \%$ and 3.3\% respectively (Toh et al., 1996). On the other hand, $S$. Entertidis was the predominated serotype in Saudi Arabia with percentage of $(55.6 \%)$ followed by $S$. Typhymurium (22.2\%) Moussa et al. (2010).

Poultry farms are Salmonella enterica serovar Typhimurium and Salmonella enterica serovar Enteritides Abd El-Gany et al. (2012).

Indicating the health hazard of poultry as a major source of $S$. Typhymurium and $S$. Entertidis as a food borne pathogens and the commonest serotype causing 
disease in human (Baggesen et al., 2000 and Aktas et al., 2007).

Antimicrobial resistance in Salmonella has received consider attention as the emergence of MDR Salmonella may result in treatment failure Yan et al. (2003).

With the emergence of antibiotics resistance bacteria presenting a serious challenge in human and veterinary medicine globally, there is an abundant of evidence showing that the antimicrobial resistance of Salmonella in the chicken supply chain is more possibly attributed to the use of antibiotics in animal husbandry (Cui et al.,2016).

Examination of samples collected from diseased and died saso chicks from different hacharies in Sharkia using SMT, 37 Salmonella isolates was isolated in an over prevalence of $12.3 \%(37 / 300)$, $21 \%$ was from liver, while $11 \%$ was from 2 ceciand $5 \%$ from yolk sac. The results of serological identification in present study detected eleven different serovars from 37 Salmonella isolates, $S$.Entertides predominated with higher percentage $(27.1 \%)$, while the remained isolates were serotyped as.

$S$. Tamale, $S$. Typhymurium, $S$. Kentucky, $S$. Apeyme, $S$. Gallinarum $S$. infantis, $S$.
Takorade, $S$. Heldberg, $S$. Bargany and $S$. Larochelle with percentages $(16.2 \%),(13.5 \%)$, $(13.5 \%), \quad(10.8 \%), \quad(5.4 \%)$, (2.7\%), (2.7\%), (2.7\%), (2.7\%), (2.7\%) respectively.

Results of antimicrobial susceptibility

testing(antibiogram of Salmonella isolates ) :

All Salmonella isolates were tested for their susceptibility to the following antimicrobial agents: gentamicin (CN), ciprofloxacin (CIP), amoxicillin- clavulanic acid (AMC), doxycycline (DO), chloramphenicol (C), erythromycin (E), sulfamethoxazole trimethoprim (SXT), cefotaxime(CTX), colitis sulfate (C.T), streptomycin (S), naiidixic acid (N.A) and rifamycin (R). High rate of susceptibility was the most common finding obtained against sulfamethoxazole trimethoprime $\quad(97.3 \%)$, streptomycin $\quad(83.8 \%)$ chloramphenicol and ciprofloxacin $(75.7 \%)$ as shown in table (8). Also, absolute resistance was obtained among Salmonella isolates against erythromycin, cefotaxime, colistin sulfate and rifamycin $(100 \%)$ for each one followed by amoxicillin clavulanic acid (75.7\%).

In particular, there are many reports of increasing prevelance of fluroquinolone -resistant 
Salmonella Piddock, (2002) and Wasyl et al., (2013) which might be a potential risk for human health.

The results of this study revealed the potential problem of widespread of multidrug resistant Salmonella species especially in chickens because of extensor use of antimicrobial agent, in human and veterinary medicine that was agree with Briggs and Fratamsco (1999) as we detect resistance to CIP was $24 \%$ that is nearly the same as Ren et al. (2016) and Nhung et al. (2018) but Cui et al. (2019) detect resistance to CIP with a higher percentage $37 \%$.

In present study, it is noticed that there is a huge increase in quinolone resistance in Salmonella that is go hand in hand with Mobalk et al. (2002). Controversially, no resistance of
Salmonella to ciprofloxacin in Egypt from broiler carcass Mona (2014) and in Brazil (Cordoso et al. (2006). The burden of food born disease is increasing due to antimicrobial resistance which represents a great risk of treatment failure, however, very little is known about the resistance profile of food born pathogen Bantawak et al. (2019)

Therefore, efforts are needed to reduce the prevalence of resistant Salmonella in broiler chickens, including the adoption of guide lines for the prudent use antimicrobial agents in animals used for food. There is a need for continued surveillance to determine a regular antimicrobial susceptibility data to detect any changing of resistance pattern Kumar et al., (2012).

Table (1): Prevalence of Salmonella serotypes among different organs of chicks (each organ represent one chick)

\begin{tabular}{|c|c|c|c|c|c|c|c|c|c|c|c|c|}
\hline \multirow[b]{2}{*}{ Organ (No) } & \multirow[b]{2}{*}{$\begin{array}{l}\text { No of } \\
\text { Salmonella } \\
\text { isolates }\end{array}$} & \multicolumn{11}{|c|}{ Salmonella serotypes(37) } \\
\hline & & 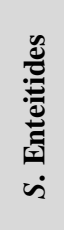 & $\begin{array}{c}\frac{0}{\widetilde{J}} \\
\stackrel{\Xi}{\tilde{n}} \\
\dot{v}\end{array}$ & 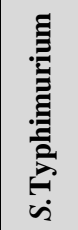 & 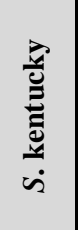 & 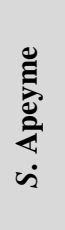 & 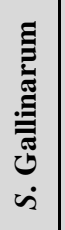 & 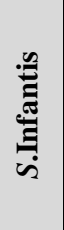 & 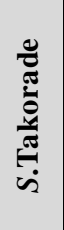 & 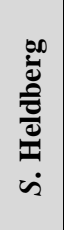 & 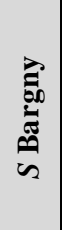 & 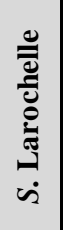 \\
\hline Liver(100) & $21 \%$ & 6 & 4 & 3 & 2 & 3 & - & 1 & 1 & - & 1 & - \\
\hline $2 \operatorname{ceci}(100)$ & $11 \%$ & 4 & 2 & 1 & 2 & 1 & - & - & - & 1 & - & - \\
\hline $\begin{array}{c}\text { Yalk } \\
\operatorname{sac}(100)\end{array}$ & $5 \%$ & - & - & 1 & - & - & 2 & - & - & - & - & 1 \\
\hline Total (300) & $\begin{array}{c}37 \\
12.3 \%\end{array}$ & $\begin{array}{c}10 \\
27.19\end{array}$ & $\begin{array}{c}6 \\
16.2 \%\end{array}$ & $\begin{array}{c}5 \\
3.5 \%\end{array}$ & $\begin{array}{c}5 \\
13.5 \%\end{array}$ & $\begin{array}{c}4 \\
0.8 \%\end{array}$ & $\begin{array}{c}2 \\
540\end{array}$ & 1 & $\begin{array}{c}1 \\
2.7 \%\end{array}$ & $\begin{array}{l}1 \\
270\end{array}$ & 1 & $\begin{array}{c}1 \\
2.7 \%\end{array}$ \\
\hline
\end{tabular}


Table (2): Species susceptibility testing to different antimicrobial discs

\begin{tabular}{|c|c|c|c|c|c|c|c|c|c|c|c|c|c|}
\hline $\begin{array}{c}\text { Code } \\
\text { no }\end{array}$ & & $\sum_{i}^{U}$ & 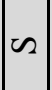 & Z & $\mathbb{Z}$ & ह & 㫐 & 武 & రু & (1) & 0 & 已 & $\frac{L}{\alpha}$ \\
\hline 22 & \multirow{10}{*}{ S.Enteritides } & - & $\mathrm{I}$ & $\mathrm{I}$ & - & - & - & $\mathrm{S}$ & I & - & $\mathrm{I}$ & - & - \\
\hline 23 & & - & $\mathrm{I}$ & $\mathrm{I}$ & - & - & - & $\mathrm{S}$ & $\mathrm{I}$ & - & $\mathrm{I}$ & - & - \\
\hline 27 & & - & $\mathrm{S}$ & $\mathrm{I}$ & - & $\mathrm{I}$ & - & $\mathrm{I}$ & $\mathrm{I}$ & - & $\mathrm{I}$ & - & - \\
\hline 36 & & - & $\mathrm{S}$ & $\mathrm{I}$ & $\mathrm{I}$ & $\mathrm{S}$ & - & $\mathrm{S}$ & - & - & $\mathrm{I}$ & -1 & - \\
\hline 38 & & - & $\mathrm{S}$ & $\mathrm{I}$ & $\mathrm{I}$ & $\mathrm{S}$ & - & $\mathrm{I}$ & - & - & - & - & - \\
\hline 42 & & - & $\mathrm{I}$ & $\mathrm{I}$ & - & - & - & $\mathrm{S}$ & I & - & $\mathrm{I}$ & - & - \\
\hline 44 & & - & $\mathrm{I}$ & $\mathrm{I}$ & - & - & - & $\mathrm{S}$ & I & - & $\mathrm{I}$ & - & - \\
\hline 92 & & - & - & - & $\mathrm{I}$ & $\mathrm{S}$ & - & $\mathrm{I}$ & - & - & $\mathrm{S}$ & - & - \\
\hline 105 & & - & - & $\mathrm{I}$ & - & $\mathrm{I}$ & - & - & $\mathrm{I}$ & - & - & - & - \\
\hline 108 & & - & $\mathrm{i}$ & - & - & $\mathrm{I}$ & - & $\mathrm{I}$ & - & - & - & - & - \\
\hline 13 & \multirow{6}{*}{$S$. Tamale } & $\mathrm{I}$ & $\mathrm{I}$ & $\mathrm{s}$ & $\mathrm{I}$ & $S$ & - & $\mathrm{I}$ & $\mathrm{I}$ & - & $\mathrm{I}$ & - & - \\
\hline 18 & & - & $\mathrm{I}$ & $\mathrm{I}$ & - & - & - & $\mathrm{S}$ & $\mathrm{I}$ & - & $\mathrm{I}$ & - & - \\
\hline 46 & & - & $\mathrm{I}$ & $S$ & $\mathrm{I}$ & $S$ & - & $\mathrm{S}$ & - & - & $\mathrm{S}$ & - & - \\
\hline 87 & & $\bar{I}$ & $\mathrm{~S}$ & $\mathrm{I}$ & - & $\mathrm{I}$ & - & $\mathrm{I}$ & - & - & $\mathrm{S}$ & - & - \\
\hline 91 & & I & $\mathrm{S}$ & $\mathrm{S}$ & $\mathrm{I}$ & $S$ & - & $\mathrm{I}$ & $\mathrm{I}$ & - & $\mathrm{I}$ & - & - \\
\hline 93 & & - & $\mathrm{S}$ & $S$ & $\mathrm{I}$ & $S$ & - & $\mathrm{I}$ & - & - & $\mathrm{I}$ & - & - \\
\hline 17 & \multirow{5}{*}{ S.Ttyphymurium } & $\mathrm{I}$ & - & - & - & $\mathrm{I}$ & - & I & - & - & - & - & - \\
\hline 20 & & - & $\mathrm{I}$ & $\mathrm{I}$ & - & - & - & $\mathrm{S}$ & $\mathrm{I}$ & - & $\mathrm{I}$ & - & - \\
\hline 21 & & - & $\mathrm{I}$ & $\mathrm{I}$ & - & - & - & $\mathrm{S}$ & $\mathrm{I}$ & - & $\mathrm{I}$ & - & - \\
\hline 26 & & - & - & - & - & $\mathrm{I}$ & - & I & - & - & - & - & - \\
\hline 31 & & - & $\mathrm{I}$ & - & - & $S$ & - & I & $\mathrm{I}$ & - & - & - & - \\
\hline 5 & \multirow{5}{*}{ S.Kentucky } & - & $\mathrm{I}$ & - & - & $\mathrm{I}$ & - & I & - & - & - & - & - \\
\hline 10 & & - & $\mathrm{I}$ & $\mathrm{I}$ & - & - & - & $S$ & $\mathrm{I}$ & - & $\mathrm{I}$ & - & - \\
\hline 11 & & - & $\mathrm{I}$ & $\mathrm{I}$ & - & $S$ & - & I & - & - & $\mathrm{S}$ & - & - \\
\hline 33 & & - & $\mathrm{I}$ & $\mathrm{I}$ & - & $\mathrm{I}$ & - & $\mathrm{S}$ & - & - & - & - & - \\
\hline 34 & & - & $\mathrm{I}$ & - & $\mathrm{I}$ & $\mathrm{I}$ & - & I & - & - & - & - & - \\
\hline 51 & \multirow{4}{*}{ S.Apeyme } & I & $\mathrm{S}$ & $\mathrm{I}$ & $\mathrm{I}$ & $S$ & - & I & $\mathrm{I}$ & - & & - & - \\
\hline 52 & & - & $\mathrm{I}$ & $\mathrm{I}$ & - & - & - & $\mathrm{S}$ & $\mathrm{I}$ & - & $\mathrm{S}$ & - & - \\
\hline 55 & & - & $\mathrm{S}$ & $\mathrm{I}$ & $\mathrm{I}$ & $\mathrm{I}$ & - & I & $\mathrm{I}$ & - & $\mathrm{I}$ & - & - \\
\hline 57 & & - & $\mathrm{S}$ & - & $\mathrm{I}$ & $S$ & - & I & $\mathrm{I}$ & - & $\mathrm{S}$ & - & - \\
\hline 77 & \multirow{2}{*}{ S.Gallinarum } & I & $\mathrm{S}$ & $\mathrm{I}$ & $\mathrm{I}$ & $S$ & - & $\mathrm{S}$ & - & - & $\mathrm{S}$ & - & - \\
\hline 79 & & $\mathrm{I}$ & $\mathrm{S}$ & $\mathrm{I}$ & $\mathrm{I}$ & $S$ & - & $\mathrm{s}$ & $\mathrm{I}$ & - & $\mathrm{S}$ & - & - \\
\hline 74 & S.Infantis & $\mathrm{I}$ & $\mathrm{S}$ & - & $\mathrm{I}$ & $\mathrm{S}$ & - & $\mathrm{S}$ & $\mathrm{I}$ & - & $\mathrm{S}$ & - & - \\
\hline 86 & S.Takorade & $\mathrm{I}$ & $\mathrm{I}$ & $\mathrm{I}$ & $\mathrm{I}$ & $\mathrm{S}$ & - & $\mathrm{I}$ & $\mathrm{I}$ & - & $\mathrm{I}$ & - & - \\
\hline 97 & S.Heldberg & - & $\mathrm{I}$ & - & - & $\mathrm{I}$ & - & I & $\mathrm{I}$ & - & $\mathrm{I}$ & - & - \\
\hline 101 & S.Bargny & - & $\mathrm{I}$ & $\mathrm{I}$ & $\mathrm{I}$ & $S$ & - & $\mathrm{I}$ & $\mathrm{I}$ & - & $\mathrm{I}$ & - & - \\
\hline 106 & S.Larochelle & - & $\mathrm{I}$ & $\mathrm{i}$ & $\mathrm{I}$ & $S$ & - & I & $\mathrm{I}$ & - & $\mathrm{I}$ & - & - \\
\hline
\end{tabular}


Table (3): antimicrobial resistance paterrn of Salmonella isolates

\begin{tabular}{|c|c|c|c|c|c|c|c|c|c|c|c|c|}
\hline Serotype & 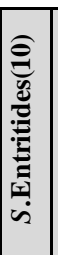 & 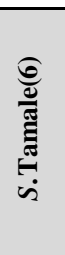 & 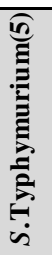 & 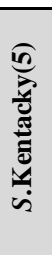 & 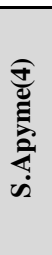 & 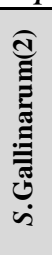 & 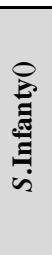 & 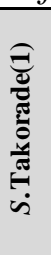 & 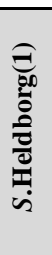 & 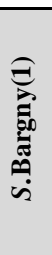 & 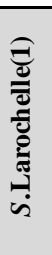 & 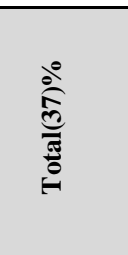 \\
\hline $\begin{array}{c}\text { Four } \\
\text { antibiotics }\end{array}$ & 0 & 2 & $\mathbf{0}$ & $\mathbf{0}$ & 1 & 1 & $\mathbf{0}$ & 1 & o & $\mathbf{0}$ & $\mathbf{0}$ & $5(13.5 \%)$ \\
\hline $\begin{array}{c}\text { Five } \\
\text { antibiotics }\end{array}$ & 0 & $\mathbf{0}$ & $\mathbf{0}$ & 0 & 1 & 1 & 1 & $\mathbf{0}$ & 0 & 1 & 1 & $5(13.5 \%)$ \\
\hline Six antibiotics & 2 & 3 & $\mathbf{0}$ & $\mathbf{0}$ & 1 & $\mathbf{0}$ & $\mathbf{0}$ & $\mathbf{0}$ & $\mathbf{0}$ & 0 & $\mathbf{0}$ & $6(16.2 \%)$ \\
\hline $\begin{array}{c}\text { Seven } \\
\text { antibiotics }\end{array}$ & 6 & 1 & 2 & 2 & 1 & $\mathbf{0}$ & $\mathbf{0}$ & 0 & 1 & $\mathbf{0}$ & $\mathbf{0}$ & $\begin{array}{c}13 \\
(3501 \%)\end{array}$ \\
\hline $\begin{array}{l}\text { More than } \\
7 \text { antibiotics }\end{array}$ & 2 & $\mathbf{0}$ & 3 & 3 & 0 & 0 & $\mathbf{0}$ & o & 0 & $\mathbf{0}$ & $\mathbf{0}$ & $8(21.6 \%)$ \\
\hline $\begin{array}{l}\text { No resistance } \\
\text { to any }\end{array}$ & 0 & 0 & $\mathbf{0}$ & 0 & $\mathbf{0}$ & 0 & 0 & o & o & o & 0 & $0(0 \%)$ \\
\hline
\end{tabular}

Table (4): Sensitivity percentage of different Salmonella species to each microbial agent:

\begin{tabular}{|c|c|c|c|c|c|c|c|c|c|c|c|c|}
\hline 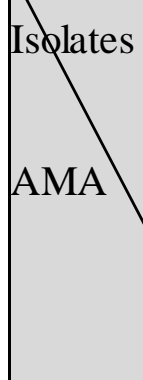 & 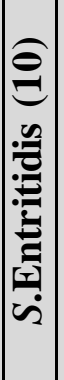 & 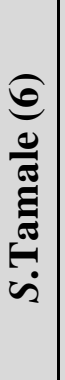 & 皇 & 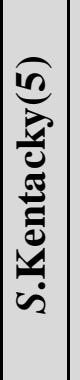 & 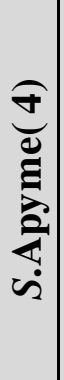 & 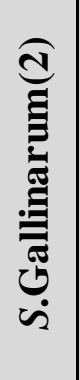 & 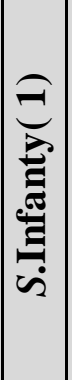 & 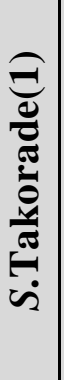 & 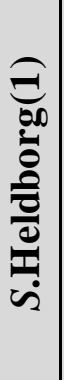 & 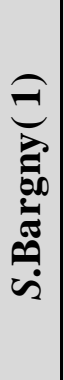 & 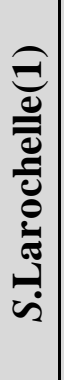 & $\begin{array}{c}\text { Total } \\
(37)\end{array}$ \\
\hline$\overline{\text { CIP }}$ & 6 & 5 & 3 & 4 & 3 & 2 & 1 & 1 & 1 & 1 & 1 & $28(7507 \%)$ \\
\hline $\mathrm{C}$ & 7 & 6 & 2 & 2 & 4 & 2 & 1 & 1 & 1 & 1 & 1 & $7 \%)$ \\
\hline $\mathrm{CN}$ & 8 & 6 & 2 & 3 & 3 & 2 & 0 & 1 & 0 & 1 & 1 & $27(72.9 \%)$ \\
\hline SXT & 9 & 6 & 5 & 5 & 4 & 2 & 1 & 1 & 1 & 1 & 1 & $36(97.3 \%)$ \\
\hline CT & 0 & 0 & 0 & 0 & 0 & 0 & 0 & 0 & 0 & 0 & 0 & $0(0 \%)$ \\
\hline CTX & 0 & 0 & 0 & 0 & 0 & 0 & 0 & 0 & 0 & 0 & 0 & $0(\%)$ \\
\hline DO & 6 & 3 & 3 & 1 & 4 & 2 & 1 & 1 & 1 & 1 & 1 & $24(64.9 \%)$ \\
\hline$S$ & 8 & 6 & 3 & 5 & 4 & 2 & 1 & 1 & 1 & 1 & 1 & $31(83.2 \%)$ \\
\hline$\overline{\mathrm{NA}}$ & 2 & 4 & 0 & 1 & 3 & 2 & 1 & 1 & 0 & 1 & 1 & $16(43.2 \%)$ \\
\hline $\mathrm{RF}$ & 0 & 0 & 0 & 0 & 0 & 0 & 0 & 0 & 0 & 0 & 0 & $0(0 \%)$ \\
\hline AMC & 0 & 3 & 1 & 0 & 1 & 2 & 1 & 1 & 0 & 0 & 0 & $9(24.3 \%)$ \\
\hline$E$ & 0 & 0 & 0 & \begin{tabular}{l|l|}
0 \\
\end{tabular} & 0 & 0 & 0 & 0 & 0 & 0 & 0 & $0(0 \%)$ \\
\hline
\end{tabular}


References

Abd El-Ghany W, El-shafii S, Hatem M. A (2012): Asurvay on Salmonella species isolated from chicken flocks in Egypt.Asian $\mathrm{J}$ Anim vet Adv.489-501.

Ahmed H. A. (2003): Tracing some sources of infection of some zoontic bacteria among family Enterobacteriaceae. M.V.Sc.Thesis, Fac. Vet. Med. Zoonoses Department, Zagazig Univerisity.

Akbarmehr J (2010): Isolation of Salmonella spp. From poultry (ostrich pigeon, and chicken) and detection of their hilA gene by PCR method. African journal of Microbiology.,4 (24): 26782681.

Aktas Z, Martin D, Kayacan C B, Diren S and Threlfall E J (2007):

Molecular characterization of Salmonella Typhimurium and Salmonella Enteritidis by plasmid analysis and pulsed-field gel electrophoresis. Inter. J. Antimicrob. Agent, 30: 541545.

Al - Shawabkeh K and Yamani, M I. (1996): Prevalence of Salmonella in poultry farms in Jordan. Pure Appl. Sci., B, 23 (1): 67 -72.

Ammar AM1, Mohamed AA, Abd El-Hamid MI, ElAzzouny MM ,(2016):
Virulence genotypes of clinical SalmonellaSerovars from broilers in Egypt. J Infect Dev Ctries. 28;10(4):337-46. doi: 10.3855/jidc.7437.

Ammar AM, Abdeen EE, Abo-Shama UH, Fekry E, Kotb EImahallawy E(2019): Molecular characterization of virulence and antibiotic resistance genes among Salmonella serovars isolated from broilers in Egypt. Lett Appl Microbiol. 2019 Feb;68(2):188-195. doi: 10.1111/lam.13106. Epub 2019 Jan 6.

Baggesen L S, Sandvang D and Aarestrup F M (2000): Characterization of Salmonella enterica serovar typhimurium DT104 Isolated from Denmark and Comparison with Isolates from Europe and the United States. J. Clin. Microbiol.,38 (4):1581-1586.

Bantawa K, Sah SN, Subba Limbu D, Subba P, Ghimire A (2019): Antibiotic resistance patterns of Staphylococcus aureus, Escherichia coli, Salmonella, Shigella and Vibrio isolated from chicken, pork, buffalo and goat meat in eastern Nepal. BMC Res Notes. 12(1):766. doi: 10.1186/s13104019-4798-7.

\section{Briggs $C E$, and Fratamsco $P$} M. (1999): Molecular characterization of an antibiotic 
resistance gene cluster of Salmonella typhimurium DT 104- antimicrob. Agents chemother., 43: 846-849.

Cardoso M O, Ribeiro A R, Santos L R, Pilotto F, Moraes H L S, Salle CTP, Rocha SLS and NascimentoV $P$ (2006): Antibiotic resistance in Salmonella enteritidis isolated from broiler carcasses. Brazil.J. Microbiol., 37: 368-371

Clinical and Laboratory Standards Institute (CLSI) (2011): Performance Standards for Antimicrobial Susceptibility Testing; Twenty-first Informational Supplement, CLSI document M100-S21, Wayne, Pennsylvania, USA, 30 (15): 42-46.

Cui M.,Xie M.,Gu Z.,Zhao S.,Wang J., and Wang Y.,(2016):prevalence and antimicrobial resistance of Salmonella isolated from integrated broiler chicken supply chain in quingdo, China.Food control .62 270276.

ISO 6579 (2002): Microbiology of food and animal feeding stuffs. Horizontal methods for the detection of Salmonella species. (4th ed.) international Organization for standardization.

$\begin{array}{llr}\text { Kauffman } & \text { F. (1973): } \\ \text { Serological } & \text { diagnosis of }\end{array}$

Salmonella spp. Kaufmann White Scheme, Copenhagen, Denmark.

Kumar T, Mahajan N K and Rakha N K (2012): Isolation and prevalence of Salmonella serovars from poultry in different parts of Haryana, India. Indian J. Anim. Sci., 82(6): 557-560.

Maripandi A and Al-Salamah A A (2010): Multiple-antibiotic resistance and plasmid profiles of Salmonella enteritidis isolated from retail chickens' meats. American Journal of Food Technology., 5 (4): 260268.

Mohammed L N, Smaha H A, Draz A A and Haggage $Y \mathbf{N}$ (1999): Salmonella among birds and human beings. Alex. J. Vet. Sic., (1): 147-154.

Moawad AAI (2009): Bacteriological studies on Salmonella Enteritidis isolated from different sources in Dakhlia Governorate. M. V. Sc. Thesis, Fac. Vet. Med., Zoonoses: Dept., Zag. University.

Molback M, Gerrner-Smidt $P$ and Wegener HC (2002) : Increasing quinolone resistance in Salmonella enterica serotype Enteritidis. Emerg. Infect. Dis., 8 (5): 514-515. 
Mona M.El-Azzouny (2014): Occurrence of Virulence Genes among Multidrug

Resistant Salmonellae Isolated from Broilers. thesis (Bacteriology), Fac. Vet. Med., Zagazig University

Moussa I M, Gassem M A, AlDoss A A, Mahmoud W A, Sadik and Abdel $M$ A L (2010): Using molecular techniques for rapid detection of Salmonella serovars in frozen chicken and chicken products collected from Riyadh, Saudi Arabia. African Journal of Biotechnology., 9 (5): 612-619.

Nhung N. T., Van N. T. B., Cuong N. V, Duong T. T., and Hang T. (2018): Antimicrobial residues and resistance against critically important antimicrobials in non typhoidal Salmonella from meat sold in Vietnam.Int.J.Food Microbe(266)301-309.

Orji M U, Onuigbo $\mathrm{H} C$ and Mbata T I (2005): Isolation of Salmonella from poultry dropping and other environmental sources in Awka, Nigeria. Int. J. Inf. Dis., 9 (2): 86-89.

Piddock L.J. (2002): Flouroquinolone resistance in Salmonella serovars isolated from human and food animals.FEMS.Microbiol.Rev.2 6 3-16.
Pieskus J, Milius J, Michalskiene I and Zagrebneviene G (2006): The distribution of Salmonella serovars in chickens and human in Lithuania. Journal of Veterinary Medicine., 53 (1): 12-16.

Ren C, Zhao Y and Shen Y. (2013): Analysis of the effect of integrons on drug-resistant Staphylococcus aureus by multiplex PCR detection.Molecular Med. Reports; 7(3): 719-724.

Sadoma A M (1997): Salmonella in chickens in connection with human infection. M. V. Sc. Thesis, Fac. Vet. Med., Tanta Univerisity

Sedeik ME, EI-Shall NA, Awad AM, Elfeky SM, Abd El-Hack ME, Hussein EOS, Alowaimer AN, Swelum AA (2019): Isolation, conventional and molecular characterization of Salmonella spp. from newly hatched broiler chicks. AMB Express. $\quad 30 ; 9(1): 136 . \quad$ doi: 10.1186/s 13568-019-0821-6.

Shah A $H$ and Korejo $\mathbf{N}$ (2012): Antimicrobial resistance profile of Salmonella serovars isolated from chicken meat. J. Vet. Anim . Sci., (2): 40-46

Taha, N A A (2002): Zoonotic importance of enter pathogenic E. coli (EPEC). $\mathrm{PhD}$ Thesis, Fac.Vet. Med. Zagazig University. 
Toh S L, Teo T P and Tay $\mathbf{Y}$ Antimicrobial resistance in H (1996): Salmonella commensal Escherichia coli serotypes isolated in Central isolated from animals at Veterinary Laboratory slaughter. Front Microbiol Aug between 1990 and 1994, 5; 4:221. doi: Singapore. Journal of $10.3389 /$ fmicb.2013.00221.

Primary Industries, 24: 67-80.

Verma J C and Gupta B R (1995): Occurrence of Salmonella serotypes in animals in India. Ind. Journal Comp. Microbiol. Immmnol. Infec. Dis., 16 (3-4): 104 - 108.

Wasyl D, Hoszowski A, Zając Yan S S, Pendrak M L, AbelaRidder B, Punderson J W, Fedorko DP and Foley $\mathrm{S}$ L (2003): An overview of Salmonella typing Public health perspectives. Clinical and Applied Immunology Reviews, 4: 189-204.

تم تجميع 300 عينه من دجاج تسمين عمر يوم يعاني العربي من مشاكل تنفسية و التهابات في

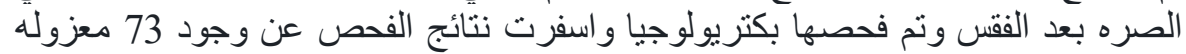

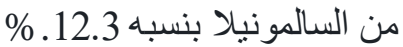
أظهرت الطرق التقليدية لعزل وتحديد معزولات السالمونيلا من الكتاكيت أن السالمونيلا مظهر مستعمر ات عديمة اللون على وسط أجار . Macconkeys أعطت خصائص لتص منطقة

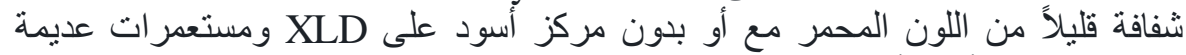

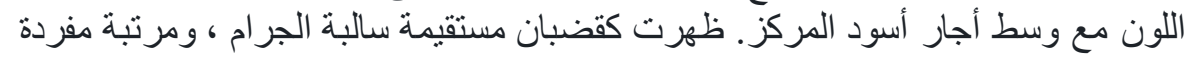

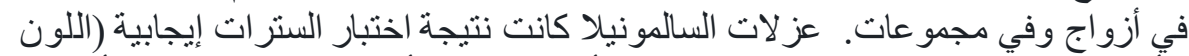

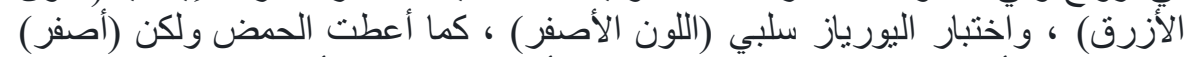

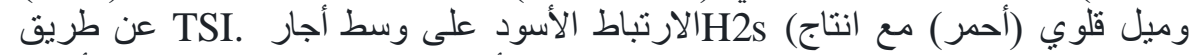

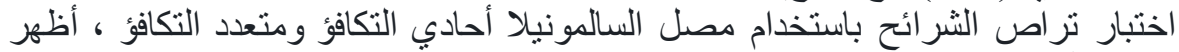

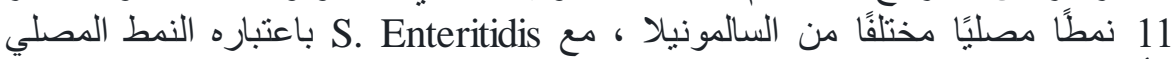

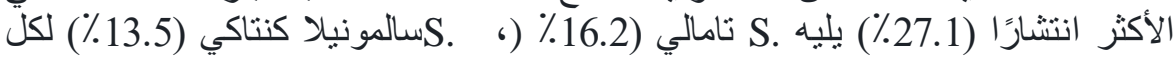

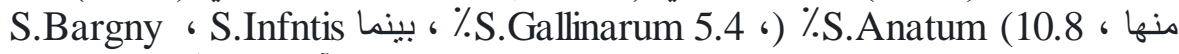

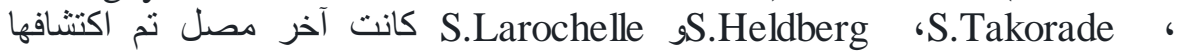

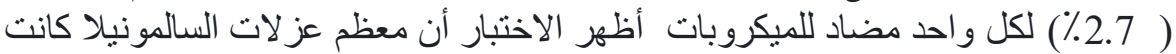

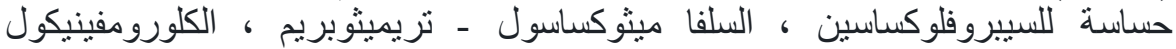

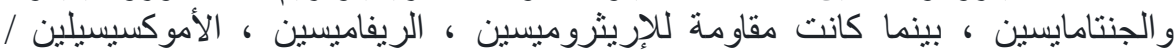

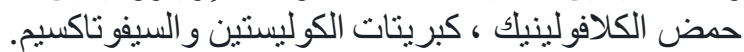

